\title{
Tendencias de la descripción bibliográfica en revistas españolas de acceso abierto
}

\author{
Rosana López Carreño \\ Francisco Javier Martínez Méndez *
}

Artículo recibido:

2 de abril de 2013.

Artículo aceptado:

3 de abril de 2013.

\section{Resumen}

El uso de normas y estilos de descripción bibliográfica estándares a nivel internacional ha dotado a las revistas científicas de un considerable nivel de rigor y uniformidad para el intercambio y manejo de datos bibliográficos entre sistemas distintos, al influir positivamente en la difusión y el impacto de la producción científica. En pleno auge de la publicación en acceso abierto creemos conveniente estudiar estos patrones de comportamiento, concretamente en las revistas editadas en España. El análisis destaca la "herencia editorial" presente en las revistas de acceso abierto que procede de las revistas impresas en la forma de citación y las normas empleadas, en donde el estilo APA es el más utilizado en la muestra objeto de estudio. No obstante,

* Ambos autores pertenecen a la Universidad de Murcia, España. (Rosana: rosanalc@ um.es); (Francisco: javima@um.es). 
el resultado global no es del todo satisfactorio debido al escaso porcentaje de uso de estas normas internacionales en las revistas españolas de acceso abierto.

Palabras clave: Revistas de Acceso Abierto; Iniciativa de Acceso Abierto; Descripción Bibliográfica; Normalización; Estilos Bibliográficos; Referencias Bibliográficas.

\section{Abstract}

Current trends in bibliographic description in Spanish open access journals

Rosana López-Carreño and Francisco-Javier MartínezMéndez

The use of international standards for bibliographic description and writing styles has imbued scientific journals with a considerable rigor and uniformity for the management and exchange of bibliographical data between diverse systems, enhancing dissemination and scientific production itself. At the current juncture of open access publishing, the study of these behavioral patterns exhibited in open access journals published in Spain seems particularly important. The analysis offered reveals the "print media legacy" evident in open access journals, embodied in APA standard citation conventions used in most journals examined in this study. Overall results, however, are not entirely satisfactory, because of the scarce use of such international standards in the Spanish open access journals.

Keywords: Open Access Journals; OAI; Bibliographic Description; Standardization; Bibliographic Styles; Bibliographic References.

\section{INTRODUCCIÓN}

$\mathrm{D}^{\mathrm{e}}$ esde hace más de tres siglos, es un hecho constatado que la Ciencia avanza gracias al "[...] acceso al conocimiento actual, a partir del cual se diseñan y ejecutan nuevas investigaciones cuyos resultados son ofrecidos de nuevo a la comunidad científica. Si los resultados de una investigación no son publicados la cadena se rompe, de manera que la comunidad pierde una 
aportación al conocimiento, a su comunicación" (Pérez Solís y Rodríguez Fernández, 2005: 61). Hasta el momento presente, y también en la actualidad, la gestión de esta comunicación científica ha sido desarrollada por editoriales científicas "[...] convertidas en propietarias de los derechos de copia y reproducción (copyright), dando lugar a un importante negocio de escala mundial" (Pérez Solís y Rodríguez Fernández, 2005: 61) al cual no todos los científicos pueden acceder ante el elevado costo de estos productos, y esto rompe la cadena de generación científica e introduce más obstáculos al desarrollo socioeconómico global. Contra esta ruptura surge la Iniciativa del Acceso Abierto a la Información (OAI), propuesta en la Declaración de Budapest de 2002 con la idea de aprovechar Internet para retirar las barreras de acceso a la literatura científica, lo que “[...] acelerará la investigación, enriquecerá la educación, compartirá el aprendizaje de los ricos con los pobres y el de los pobres con el de los ricos, hará esta literatura tan útil como sea posible y sentará los cimientos para unir a la humanidad en una conversación intelectual común y búsqueda del conocimiento" (Iniciativa de Budapest..., 2002: s. pág.).

Hace ya algún tiempo que esta propuesta es una realidad en el ámbito de la comunicación científica, a pesar de la resistencia de las editoriales privadas especializadas a que se publiquen y distribuyan revistas científicas apoyándose también para ello en la actual tendencia de consultar las revistas en formato electrónico. Laakso et al. analizaron la evolución del número de revistas en acceso abierto y del número de artículo publicados en ellas, y concluyeron que "[...] los resultados apuestan por la sostenibilidad del acceso abierto como una forma de comunicación científica, con un gran número de las revistas originales todavía en activo y con mayor número medio de artículos por revista y año superior que las publicadas en revista de pago" (2011: 7). Hoy en día, esta iniciativa es más una realidad contrastada que un proyecto o propuesta, afortunadamente.

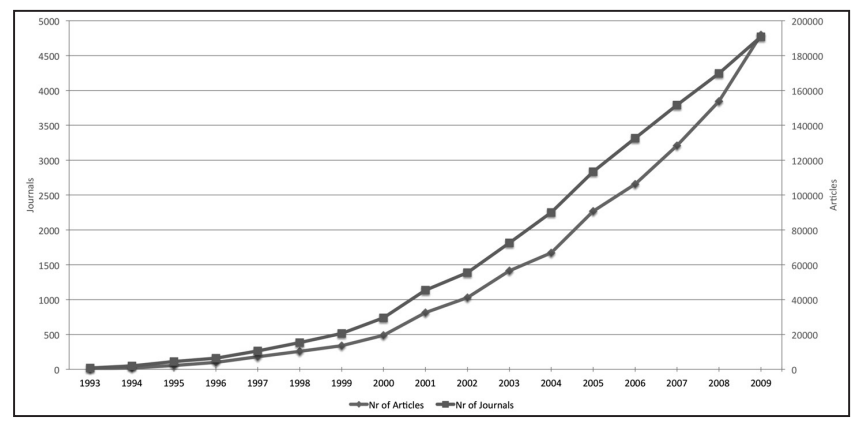

Figura 1. Evolución de las revistas científicas en OAl y del número de artículo publicados. (Laakso et al., 2011: 6) 
Al mismo tiempo que crece su número, las publicaciones científicas que han optado por el acceso abierto van afianzando de forma paulatina sus niveles de calidad y aumentando su impacto dentro de la globalidad de todas las revistas científicas. Además del grado de concienciación de los autores, que disponen libremente de su propiedad intelectual sin necesidad de cederla a intermediarios para llevar a cabo la publicación de sus trabajos, resultan determinantes

[...] las grandes barreras económicas impuestas por los monopolios editoriales, que incluyen aumento de precios de suscripciones, la pérdida del acceso por parte de los científicos a sus propios trabajos, y el avance y aceptación de las nuevas tecnologías e Internet. (Solaro y Lamberti, 2011: 2)

Buena parte de este éxito reside en la labor desarrollada por los comités editoriales de estas publicaciones, que reproducen en los procesos de revisión, publicación y difusión de contenidos los mismos procedimientos y controles de calidad que son empleados habitualmente por las editoriales privadas. Los usuarios no sólo pueden consultar los artículos de las revistas de forma gratuita sino que, además, pueden descargarlos, copiarlos, imprimirlos, distribuirlos, etc. En definitiva, "[...] se trata de un modelo de difusión del conocimiento científico que supone una transformación radical en el funcionamiento de la comunicación científica" (Abadal, 2012: s. pág.). Todo esto se lleva a cabo, generalmente, de forma altruista y es llevado a cabo por comunidades de investigadores, académicos y expertos que dedican parte de su tiempo a contribuir a la mejora de estos nuevos canales de comunicación científica. Con ello se consigue una alta disponibilidad, libre y gratuita, de los contenidos científicos de alta calidad en Internet (fundamentalmente artículos de revista aunque también hay informes, tesis y otros documentos científicos) que expanden el alcance y uso de todos estos trabajos.

Las primeras revistas científicas en acceso abierto aparecieron a principios de 1990 con "[...] la creación de Surfaces y Psycoloquy, por parte de Jean-Claude Guédon y Stevan Harnad, respectivamente [...] pero el impulso fundamental se produjo en 2003 con la aparición de las primeras revistas creadas por Public Library of Science (PLoS), en especial la primera de ellas, PLOS Biology, que proporcionó una gran visibilidad al sector" (Abadal, 2012: s. pág.). No se trata de un modelo de publicación y distribución de contenidos uniforme. En algunos casos el editor restringe el acceso a los documentos unos meses (embargo), en otros hay publicaciones donde los autores mantienen el copyright de sus trabajos y pagan porque éstos sean de acceso libre y, lo que es más frecuente, están las publicaciones de dominio público en las que el autor no paga por la publicación, y mucho menos el lector (Solaro 
y Lamberti, 2011); es lo que Harnad et al. (2008) denominan "acceso abierto dorado", aunque también existe el "acceso abierto verde", consistente en que el autor publica en una revista científica (que no tiene por qué ser de acceso abierto) y a continuación publica una copia de libre acceso en un repositorio.

Muy relacionado con este último aspecto está el modelo de negocio que permite la financiación de estas publicaciones. Sobre este particular, Ruiz Pérez analizó una muestra de 2838 revistas recogidas en el directorio DOAJ ${ }^{1}$ en las que concluye que coexisten diversos modelos de negocio, algunos de ellos heredados de la tradición de la revista impresa, y en relación con los editores

[...] hay una cierta polarización. Por un lado hay un grupo pequeño de editores que cuenta con la mayoría de las revistas y/o artículos. En el otro extremo hay una mayoría, en torno al 90 \% del total de editores, que tiene tan sólo una revista. En el centro encontramos bastante variedad de opciones. (Ruiz-Pérez, 2010: 47)

Björk et al. (2010) identificaron que la distribución de áreas temáticas de las revistas científicas publicadas en acceso abierto varía en función de si se trata de "acceso verde" o "acceso dorado". En el primer grupo son mayoritarias las revistas de Ciencias de la Tierra, Física y Matemáticas (más de la mitad), mientras que con acceso abierto inmediato, las principales áreas temáticas de las revistas son la Medicina, la Bioquímica, la Genética y la Biología Molecular, junto con otras disciplinas relacionadas con la Medicina.

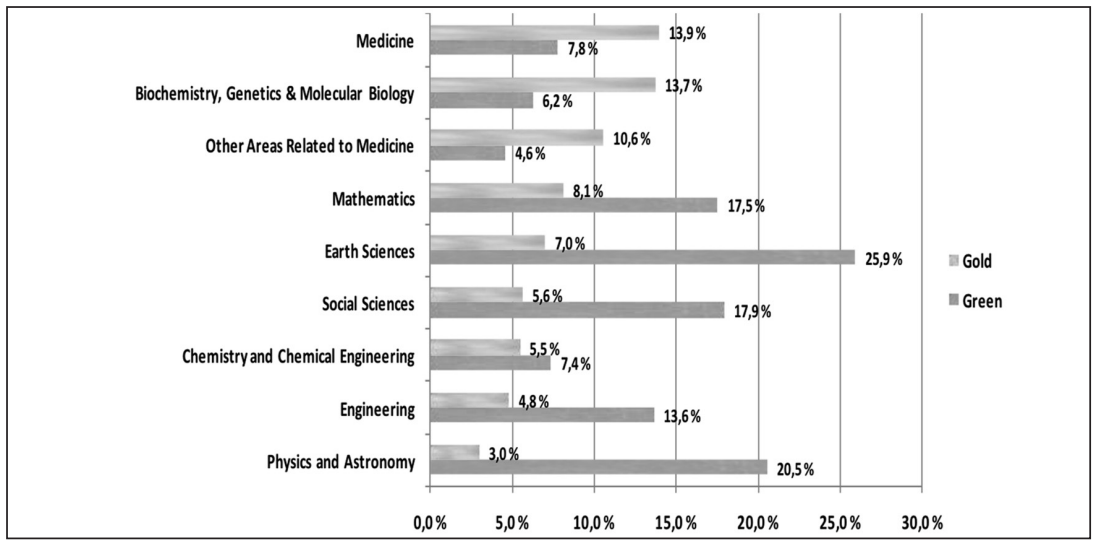

Figura 2. Distribución de los temas de las revistas publicadas en acceso abierto. (Björk et al., 2010: 8)

1 DOAJ son las siglas del Directory of Open Access Journals: directorio de revistas de acceso libre, científicas y académicas, que cumplen con estándares de alta calidad al utilizar la revisión por pares o control de calidad editorial y que son gratuitas para todos al momento de su publicación, con base en la Declaración de Budapest. 
La presencia de las revistas publicadas en acceso abierto en los índices tradicionales basados en citas, que ordenan las revistas en virtud de su factor de impacto, fue estudiada también por Ruiz Pérez (2010) quien estableció que en 2009 sólo el 38 \% de las publicaciones en acceso abierto fue recopilado por la base de datos Scopus, y que su presencia fue mucho menor en las bases de datos SCI (Science Citation Index) y SSCI (Social Science Citation Index) con un $8 \%$ en 2008. Es un hecho evidente que si bien esta presencia parece ir aumentando, no es aún excesivamente destacada y debe ser analizada con más profundidad. Este alejamiento inicial existente entre los índices de citas y la publicación en acceso abierto ha motivado, sin duda alguna, que cada vez se publiquen más trabajos sobre la necesidad de introducir nuevos indicadores de calidad para estas publicaciones, de forma suplementaria al tradicional factor de impacto (Ostrowska, 2009; Hernández Pina y Maquilón Sánchez, 2010). Obviamente éste es un tema controvertido y que precisa de mucho análisis, además de algún tiempo más para observarlo con mayor perspectiva. En un reciente trabajo, tras estudiar la correlación entre los índices basados en citas y los criterios de calidad que se siguen para evaluar una revista en Latindex, Gámez demuestra que "[...] los criterios basados en el recuento de citas no miden de la misma forma la calidad de las revistas científicas de Psicología del ámbito iberoamericano que otros criterios centrados más directamente en la calidad del proceso editorial" (2013:21).

El uso de Internet como medio para la comunicación de los artículos ha conllevado la creación de vínculos muy estrechos entre las comunidades científicas editoras y las comunidades promotoras del uso de software libre, donde el Public Knowledge Project (1998) es el ejemplo paradigmático (son los creadores del sistema de gestión y publicación de revistas científicas OJS). ${ }^{2}$ También se están consolidando los repositorios digitales científicos (como $\operatorname{arXiv}^{3}$ o PubMED ${ }^{4}$ ), en la asunción de criterios de calidad similares a los empleados por las revistas científicas frente al tradicional almacén de documentos de autoarchivo, y se ha llegado a proponer la "[...] definición de un sistema que permita evaluar y certificar la calidad de los repositorios

2 OJS son las siglas de Open Journal Systems, el software libre más empleado en la gestión y edición de las publicaciones científicas en OAI (Acceso Abierto a la Información) a nivel mundial.

3 arXiv es el principal sitio en línea de preprints de artículos científicos en el campo de las matemáticas, física, informática y biología. En muchos campos de las matemáticas y la física casi todos los artículos científicos se publican en arXiv (algunos autores prefieren usar el término eprint para denominar a estas publicaciones porque ya no se trata de borradores sino de trabajos finalizados).

4 PubMed es un recurso que permite búsquedas entre 15 millones de registros de unas 4600 revistas indizadas por MEDLINE y las referencias de OLDMEDLINE desde 1953. PubMed forma parte del sistema Entrez diseñado por el National Center for Biotechnology Information de USA para integrar la información bibliográfica y factual de interés en Ciencias de la Salud. 
según criterios internacionales [lo cual] es vital para mejorar la calidad de sus servicios, para incrementar su estandarización e interoperabilidad y para promover la visibilidad y aceptación de los repositorios como vía para la comunicación científica” (Recolecta, 2010: 3). En la implementación de estos repositorios digitales se usa de forma muy extendida el software libre. Según los datos de Valencia Velázquez (2013), dSpace ${ }^{5}$ es el software libre más utilizado en colecciones digitales, con un $40.7 \%$ de frecuencia de uso, siguiéndolo EPrints ${ }^{6}$ con un $14.2 \%$, si bien el autor ha llegado a verificar la presencia de hasta 120 paquetes de software implementados para este uso, lo que demuestra el alto índice de vinculación entre el acceso abierto y las comunidades que desarrollan software para la red que lo soporta.

Una muestra final de la importancia de este movimiento de acceso abierto a la información científica es el interés que ha demostrado Google en tener debidamente registrada esta producción y disponer del impacto de la misma en términos de citas recibidas gracias a los proyectos Google Scholar (Noruzi, 2005), Google Citation Index (Jacso, 2005) y, más recientemente, Google Journal Rankings (Kovalchik, 2012).

En España, este movimiento también se ha dejado notar. Según Abadal (2012) el 26.32 \% de las revistas científicas españolas son de acceso abierto (en algunas áreas como es el caso de la Biblioteconomía y Documentación ese porcentaje es mucho más elevado). Para Abadal (2012) este porcentaje se debe al origen de las editoriales donde predominan entidades sin ánimo de lucro, como universidades, centros públicos, sociedades científicas y asociaciones y colegios profesionales. Según datos actualizados a diciembre de 2012 y extraídos del directorio DOAJ, España ocupa el quinto lugar -en términos absolutos- en el ranking de países con revistas en acceso abierto por detrás de USA, Brasil, UK e India, lo que supone un dato relevante en el ámbito editorial científico (debe considerarse que todos estos países tienen mayor población que España así que, en términos relativos por número de habitantes, ocuparía una posición incluso más alta). La mayoría de estas revistas científicas son versiones electrónicas de títulos impresos o, en muchos casos, son ediciones que sustituyen a la edición impresa por el ahorro que supone la edición electrónica. Asimismo, gran parte de estas revistas están vinculadas a organizaciones sin ánimo de lucro que encuentran en el acceso

5 Software de código abierto desarrollado por el Instituto Tecnológico de Massachussets y Hewlett-Packard. Provee herramientas para la administración de colecciones digitales y su uso más común es como soporte de repositorios institucionales. Soporta una gran variedad de formatos de ficheros.

6 Software gratuito y de código abierto para la creación de repositorios digitales de acceso abierto. Es desarrollado por la School of Electronics and Computer Science de la Universidad de Southampton. 
abierto y en el mundo digital su hábitat comunicativo. De alguna manera, el acceso abierto en estas publicaciones electrónicas recibe una "herencia editorial" perceptible en la imagen de marca, la línea editorial, los estilos formales de redacción, los formatos de presentación de los trabajos, etc. Dentro de estas recomendaciones para la presentación de los manuscritos, poseen especial importancia los aspectos relacionados con la normalización bibliográfica, algo que debe seguir respetándose en el entorno del acceso abierto, ya que "[...] la normalización de publicaciones periódicas cobra sentido en la transferencia de información científica y comunicación de resultados de la investigación" (Delgado López-Cózar, 1997b: 39). Sería un error pensar que la normalización de las referencias bibliográficas puede relajarse, e incluso abandonarse, en el contexto de la revista electrónica en Internet, básicamente por dos motivos: 1. no hay razón alguna para ello en función de la adaptación de la revista al nuevo medio y 2 . esto dificultaría o retrasaría la inclusión de estos artículos científicos en repositorios digitales científicos, que precisan de esta normalización para lograr una "[...] fuerte repercusión en el asiento, intercambio y recuperación de información según lo recomendado por la OAI-PMH, ${ }^{7}$ lo que desde luego dará una mayor visibilidad a los trabajos académicos que se hospeden en los repositorios universitarios" (Zurita, Cervantes y Miranda, 2007: 13).

En esa interoperabilidad de los metadatos las referencias bibliográficas desempeñan un importante papel, pues resulta más efectiva esa recolección automática cuando los autores de los artículos y los editores de las revistas científicas en acceso abierto exigen el cumplimiento de normas y estilos reconocidos internacionalmente, tal como ocurre con las referencias creadas por los gestores bibliográficos tipo Zotero ${ }^{8}$ o Reference Manager ${ }^{9}$ (NISO, 2004: 4) y la disminución de tiempo que esto supone para la divulgación del contenido del documento si se siguen estas normas: "[...] cuando las referencias bibliográficas responden fielmente a una norma, su inclusión en las bases de datos electrónicas se realiza mediante un proceso automático de marcación de sus elementos. El empleo de esa facilidad reduce de forma significativa el tiempo de marcación de un artículo, y permite la generación automática de conexiones con otros textos, lo que abarata los costes y reduce el número de errores de transcripción" (Culebras Fernández, 2010: 82).

7 Es el protocolo de la Iniciativa de Acceso Abierto para la recolección de metadatos. Consiste en un mecanismo para la interoperabilidad de repositorios.

8 Software de código abierto que permite a los usuarios recolectar, administrar y citar investigaciones de todo tipo de orígenes desde el navegador web. Disponible inicialmente como complemento para el programa Firefox, también funciona como aplicación independiente.

9 Es otro gestor bibliográfico comercializado por Thomson Reuters. Puede considerarse el pionero dentro de esta línea de aplicaciones ya que el inicio de su desarrollo data del año 1982. 
Consideramos, por tanto, interesante y oportuno analizar los procedimientos de descripción bibliográfica presentes en estas revistas electrónicas en acceso abierto para determinar "[...] la exhaustividad con que se ha desarrollado un trabajo científico, para conocer cuán avanzado está un tema en el mundo, para identificar fuentes relacionadas o autores de impacto en una materia dada" (Pérez-Borges, 2010: 186), y su normalización podría ser objeto de instrumentación para la visibilidad del trabajo a través de las herramientas de vigilancia desarrolladas en torno a la producción científica. La normalización internacional de las referencias bibliográficas, además de mejorar la difusión y la comunicación de la ciencia, puede facilitar el intercambio de metadatos entre sistemas en la Web Semántica (Angrosh, Cranefield y Stanger, 2011; Benz et al., 2011) y ayudar, además, a focalizar al autor como unidad de medida (a partir de parámetros como el índice $h$, por ejemplo), complementando a la revista en la medición de su impacto.

Del mismo modo que ocurre en las revistas científicas clásicas, existen una serie de normas de mayor aplicación en las revistas en acceso abierto (ISO, APA, Vancouver, etc.) y “[...] otras creadas por bibliotecas o instituciones con la intención de establecer procedimientos uniformes de referencia para sus publicaciones o para ser empleados por los miembros y usuarios de esa organización” (Sorli Rojo y Merlo Vega, 2000: 1). Vamos a continuación a revisar su uso y aplicación en este contexto.

\section{Estilos}

Los estilos científicos son estilos de edición, es decir: “[...] modos de estructurar los contenidos, forma de redactar los artículos científicos, la presentación, organización del contenido, formas de hacer abreviaturas, de presentar cuadros y documentos adjuntos y, además, formas de hacer las citas bibliográficas y listas de referencias. Por eso las citas bibliográficas dependen de cada estilo científico" (Álvarez De Toledo, 2012). A continuación se describen brevemente los estilos presentes en las revistas de acceso abierto españolas analizadas:

a) Modern Language Associaton o MLA style: estilo muy utilizado en Humanidades, especialmente en Lengua y Literatura, y adoptado por más de 1000 revistas en todo el mundo. Acceso a normas no gratuito. 


\section{MLA style}

López Carreño, Rosana y Juan Antonio Pastor Sánchez. «Actualización del modelo de portal periodístico.» Anales de Documentación 13 (2010): 177-184.

Figura 3. Ejemplo de referencia bibliográfica siguiendo el estilo MLA.

b) The Chicago Manual of Style (CMS o CMOS): “[...] uno de los estilos bibliográficos más antiguos y más conocidos. El CMS es toda una normativa de estilo de edición, las recomendaciones tratan de puntuaciones, organización del texto, pies de página, notas, citas. El Manual se publicó por primera vez en 1903 con la intención de unificar los criterios de citas bibliográficas de los investigadores. Usado principalmente en las materias de historia, ciencias sociales, arte, musicología y literatura" (Álvarez De Toledo, 2012: s. pág.). Acceso a normas gratuito.

\footnotetext{
Chicago style

López Carreño, Rosana, y Juan Antonio Pastor Sánchez. «Actualización del modelo de portal periodístico.» Editado por Universidad de Murcia, Servicio de Publicaciones Murcia. Anales de Documentación 13 (2010): 177-184.
}

Figura 4. Ejemplo de referencia bibliográfica siguiendo el estilo Chicago.

c) APA: “[...] el estilo de citas bibliográficas oficial de la American Psychological Association y establece el formato para todo tipo de citas y documentos en Psicología y Ciencias Sociales. Creado en 1929 contempla la estructura de los documentos, longitud, puntuación, abreviaturas, cuadros, partes del manuscrito y citas bibliográficas" (Álvarez De Toledo, 2012: s. pág.). Es predominante su uso en Ciencias de la Actividad Física y el Deporte (Villamón, Devís y Valenciano, 2006). Acceso a normas gratuito en una versión reducida.

\section{APA style}

López Carreño, R., \& Pastor Sánchez, J. A. (2010). Actualización del modelo de portal periodístico. (U. d. Murcia, Ed.) Anales de Documentación, 13, 177-184.

Figura 5. Ejemplo de referencia bibliográfica siguiendo el estilo APA.

d) ASA: estilo de la American Sociological Association, basado en el Estilo Chicago. Acceso a normas no gratuito.

e) CSE: “[...] estilo originalmente llamado CBE que fue creado por el Council of Biology Editors, que actualmente se llama Council of Science Editors por lo que ahora el estilo es conocido como CSE Style. Es un estilo utilizado principalmente en Biología pero igual- 
mente en Biomedicina, Bioquímica y otras disciplinas científicas afines" (Álvarez De Toledo, 2012: s. pág.). Acceso a normas no gratuito.

f) ISO 690 e ISO 690-2: normas internacionales que establecen los criterios a seguir para la elaboración de referencias bibliográficas. En ellas se establece el orden de los elementos de la referencia bibliográfica y las convenciones para la transcripción y presentación de la información. La ISO-690-2 está destinada para documentos electrónicos. Acceso a normas no gratuito. ${ }^{10}$

g) Vancouver (UMR): “[...] creado en 1978, es el estilo establecido por el International Committee of Medical Journal Editors (ICMJE). Es conocido igualmente por Uniform Requirements for Manuscripts Submitted to Biomedical Journals o simplemente por Uniform Requirements o por URM. Es una norma de la ANSI y como tal es usado por las principales revistas de Medicina, además de la National Library of Medicine (NLM) desde 1979 y por PubMed [...] cada referencia utilizada tiene un número y las citas tienen que ir numeradas, el número es obligatorio en el contexto de la cita. Los títulos de publicaciones periódicas deben citarse en su forma abreviada, siguiendo las abreviaturas del PubMed Journal Database" (Álvarez De Toledo, 2012: s. pág.). Acceso a normas gratuito.

h) Harvard: “[...] más que un estilo bibliográfico, es una forma de realizar las citas en contexto y de hacer las correspondientes listas de referencias. Tiene su origen en 1881 en la Universidad de Harvard. Su principal característica es la utilización de las citas en contexto con el formato autor-fecha: (autor, fecha) o autor (fecha), dependiendo de los casos. La lista de referencias se hace al final del documento colocando los autores por orden alfabético y en caso de que un autor tenga diferentes citas, sus referencias van por orden cronológico. Esta forma de hacer las citas se aplicó a la mayoría de los posteriores estilos bibliográficos como MLA y APA” (Álvarez De Toledo, 2012: s. pág.). Acceso a normas gratuito.

10 Según Delgado López-Cózar (1997b: s. pág.): "ISO debería tener en cuenta que a diferencia de otros sectores donde las normas son un prerrequisito para la producción, en el ámbito documental no ocurre lo mismo [...]. Se deberían abaratar los precios de las normas e impulsar su máxima difusión a través de las propias revistas científicas. En este sentido, la actuación del Grupo Vancouver es modélica: marca el ejemplo a seguir. Los requisitos uniformes para preparar los manuscritos enviados a revistas biomédicas, elaborados por el ICMJE, no están protegidos por derechos de autor, pudiéndose copiar o reimprimir sin autorización, siempre y cuando se haga sin fines de lucro y se cite la fuente. Además, el comité alienta explícitamente a la distribución del documento por cualquier medio y pide a las revistas que deseen seguir sus recomendaciones que lo indiquen en sus instrucciones a autores. Los resultados de esta política no pueden ser más alentadores pues hoy más de 500 revistas han aceptado recibir originales preparados según dichos requisitos y su uso por los investigadores de biomedicina es bastante generalizado". 


\section{OBJETIVOS Y MÉTODO DE TRABAJO}

El presente trabajo tiene como objetivo general calibrar el nivel de uso de las normas y estándares de descripción bibliográfica internacionales en las revistas españolas publicadas en acceso abierto, y analizar el grado de adecuación de estas publicaciones a los estándares más reconocidos a nivel internacional para la comunicación científica. La hipótesis a contrastar es que las revistas científicas electrónicas en acceso abierto han adoptado el uso generalizado de las normas de descripción bibliográfica internacionales, al igual que en el pasado lo hicieron las revistas científicas impresas.

El método de trabajo se basa en lo siguiente:

1. El conjunto de publicaciones analizadas, un total de 430, se corresponde con las revistas españolas en acceso abierto recopiladas en el directorio DOAJ en diciembre de 2012. Este directorio se eligió al ser la fuente de referencia de archivos de acceso abierto en Internet que recopila revistas científicas y académicas que cumplan con estándares de alta calidad al utilizar la revisión por pares o control de calidad editorial y que eran gratuitas para todos al momento de su publicación. Quedaron por tanto excluidas de este estudio todas aquellas publicaciones que impusieron algún tipo de embargo al acceso a los contenidos o que, por razones editoriales, no se hayan registrado en este directorio.

2. Del inicial conjunto de 430 publicaciones se seleccionaron para nuestro estudio todas aquellas que indicaran explícitamente el estilo de citación y de referencias en el apartado de "Instrucciones para autores".

El periodo de recolección de datos estuvo comprendido entre el 12 y el 20 de diciembre de 2012. De cada una de esas revistas se recogió la siguiente información:

- Datos: nombre de revista, materia, formato de citación, estilo de referencia.

- Para elegir la materia (o área temática) se accedió a la sección de "Instrucciones para autores" de cada revista para la recolección de datos y, posteriormente, se clasificó en su apartado correspondiente. Las materias y submaterias inicialmente empleadas fueron las establecidas por la base de datos Dialnet ${ }^{11}$ (se eligió esta clasificación de materias al 
considerar que se aproximaba más a las áreas temáticas establecidas en el ámbito académico).

Anales de Documentación I Ciencias sociales I Información. Documentación (Ciencias sociales) | Apellidos, año, página I ISo 690:2010

Figura 6. Formato de ficha de recolección de una revista científica.

La lista inicial fue posteriormente reducida con la idea de elaborar una clasificación de materias menos dispersa que la que ofrece Dialnet (así, Artes se ha unido a Humanidades, e igualmente, se han unido las Ciencias Biológicas con Geociencias y Medio Ambiente, formando el tercero Biología, Geociencias y Medio Ambiente, tal como se puede ver en la Tabla I).

\section{Resultados}

\section{Revistas con instrucciones de formato}

Sólo 137 de las 430 revistas analizadas (aproximadamente un 32 \%) indican el formato a seguir al momento de insertar una cita en una referencia bibliográfica. Esto, además de confundir a los autores, puede terminar induciéndolos a publicar en otro sitio, pensando que la calidad de la publicación científica no sea la más óptima. Otra posible explicación a este escaso porcentaje podría ser que los editores prefieran ser ellos quienes introduzcan el formato a las referencias bibliográficas, dejando que el autor las aporte como él esté acostumbrado. En ambos casos, esto no es lo tradicional en el mundo de la publicación impresa, por lo que creemos que no es conveniente mantener más esta situación y que se debería ir corrigiendo.

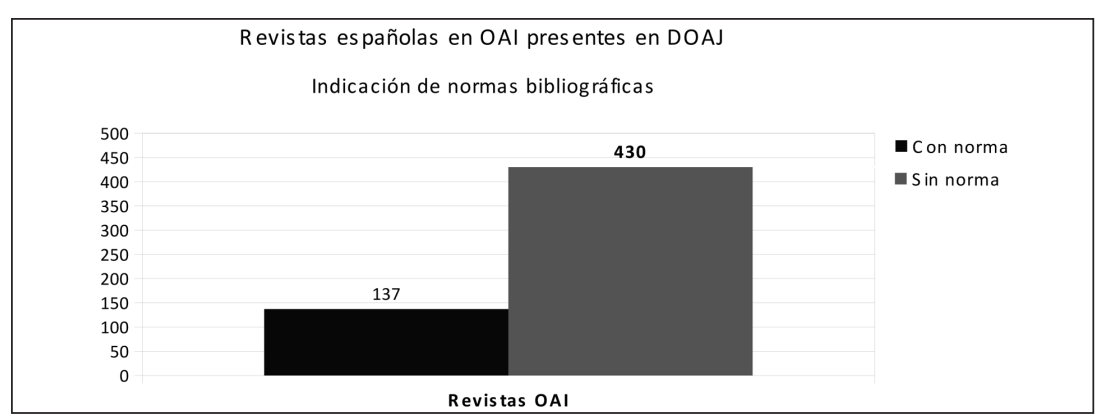

Figura 7. Distribución de revistas con normas de formato y de revistas sin norma. 
La Tabla I recoge la distribución de las revistas por materia. Se observa un predominio de las publicaciones de Psicología y Educación, de Ciencias de la Salud y de Ciencias Sociales en general.

Tabla I. Distribución por materias de las revistas españolas en OAI.

\begin{tabular}{|l|c|c|}
\hline \multicolumn{1}{|c|}{ Materia } & Núm. & $\%$ \\
\hline Arte y Humanidades & 10 & 7.30 \\
\hline Biología, Geociencias y Medio Ambiente & 7 & 5.11 \\
\hline Ciencias de la Salud & 30 & 21.90 \\
\hline Ciencias Jurídicas & 4 & 2.92 \\
\hline Ciencias Sociales & 28 & 20.43 \\
\hline Economía y Empresa & 9 & 6.57 \\
\hline Filologías & 6 & 4.38 \\
\hline Psicología y Educación & 40 & 29.20 \\
\hline Tecnologías & 3 & 2.19 \\
\hline \multicolumn{1}{|c|}{ Total } & 137 & 100 \\
\hline
\end{tabular}

\section{Presencia en Latindex y en Web of Science (WoS)}

El total de revistas españolas presentes en la base de datos Latindex fue de 152 (enero de 2013). Un total de 51 eran de acceso abierto, estaban incluidas en el DOAJ y tenían indicación de las normas de referencia bibliográfica que debían emplear.

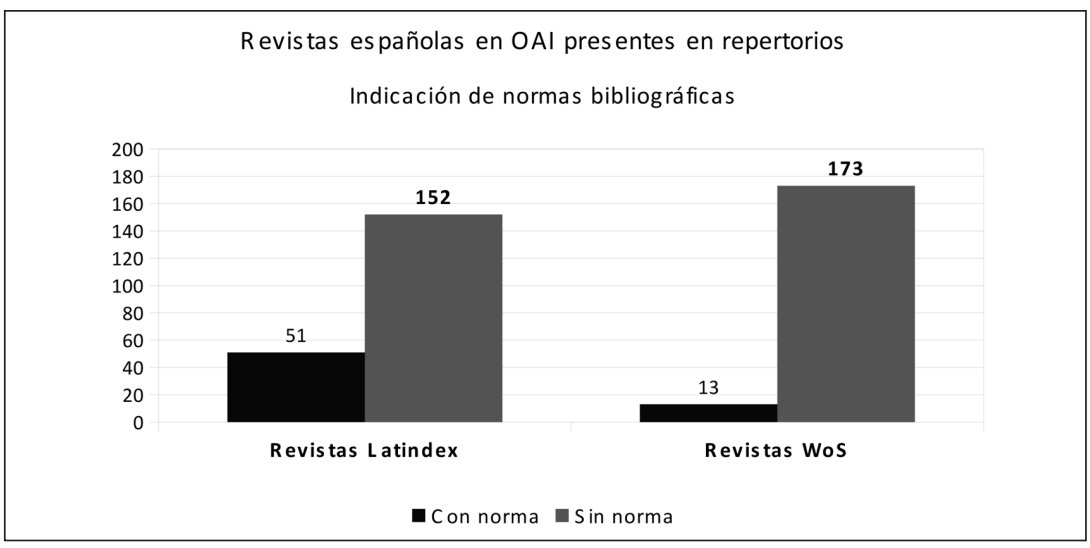

Figura 8. Distribución de las revistas españolas con presencia en Latindex y en WoS según la indicación de la norma bibliográfica recomendada.

La cifra de revistas españolas indexadas en WoS durante 2011 fue de 173, entre las que se encontraban 51 de acceso abierto, de las cuales sólo 13 tenían 
indicación de normas de estilo bibliográfico. En el primer caso el porcentaje fue más o menos similar que cuando se analizaron las revistas en su globalidad ( $33 \%$ frente a un $32 \%$ ), pero en el segundo caso, las revistas indexadas en WoS, el porcentaje descendió enormemente a menos de un $10 \%$. Por tanto, el hecho de que la revista exija un formato para la referencia bibliográfica no parece, en ambos casos, un criterio determinante para la inclusión de la publicación en estos directorios; de hecho, entre los criterios de calidad de ISI para seleccionar revistas se contempla que la información bibliográfica sea completa y de calidad, aunque no se muestre especial preocupación por los errores en las mismas ya que los más perjudicados, potencialmente, son los propios autores y las revistas que los publican (Ruiz Pérez, Delgado López-Cózar y Jiménez Contreras, 2006).

\section{Estilos de referencia bibliográfica utilizados}

En la Tabla II se puede consultar el resultado del análisis de los estilos de referencia utilizados en las 137 revistas analizadas, agrupados por cada uno de ellos. Se indica también la materia de las revistas.

Tabla II. Estilo de referencia bibliográfica empleado en las revistas españolas OAI analizadas con indicación del área de conocimiento de la publicación.

\begin{tabular}{|c|c|c|}
\hline Estilo & Materia & Núm. \\
\hline \multirow[t]{8}{*}{ APA } & Arte & 1 \\
\hline & Ciencias de la Salud & 2 \\
\hline & Ciencias Sociales & 8 \\
\hline & Economía y Empresa & 3 \\
\hline & Filologías & 3 \\
\hline & Humanidades & 1 \\
\hline & Psicología y Educación & 37 \\
\hline & Tecnologías & 2 \\
\hline APA & Total & 57 \\
\hline ASA & Ciencias Sociales & 2 \\
\hline CSE & Humanidades & 1 \\
\hline \multirow[t]{8}{*}{ Harvard } & Arte & 1 \\
\hline & $\begin{array}{l}\text { Biología, Geociencias y } \\
\text { Medio Ambiente }\end{array}$ & 3 \\
\hline & Ciencias Jurídicas & 1 \\
\hline & Ciencias Sociales & 3 \\
\hline & Economía y Empresa & 2 \\
\hline & Filologías & 1 \\
\hline & Humanidades & 1 \\
\hline & Psicología y Educación & 1 \\
\hline
\end{tabular}




\begin{tabular}{|c|c|c|}
\hline Harvard & Total & 13 \\
\hline \multirow[t]{8}{*}{ ISO 690/ISO 690-2 } & $\begin{array}{l}\text { Biología, Geociencias y } \\
\text { Medio Ambiente }\end{array}$ & 3 \\
\hline & Ciencias Jurídicas & 2 \\
\hline & Ciencias Sociales & 11 \\
\hline & Economía y Empresa & 4 \\
\hline & Filologías & 1 \\
\hline & Humanidades & 3 \\
\hline & Psicología y Educación & 1 \\
\hline & Tecnologías & 1 \\
\hline IS0 690/IS0 690-2 & Total & 26 \\
\hline \multirow[t]{4}{*}{ MLA } & Filologías & 1 \\
\hline & $\begin{array}{l}\text { Biología, Geociencias y } \\
\text { Medio Ambiente }\end{array}$ & 1 \\
\hline & Ciencias Sociales & 3 \\
\hline & Humanidades & 1 \\
\hline MLA & Total & 6 \\
\hline \multirow{3}{*}{$\begin{array}{l}\text { The Chicago Manual of } \\
\text { Style }\end{array}$} & Ciencias Jurídicas & 1 \\
\hline & Ciencias Sociales & 1 \\
\hline & Humanidades & 1 \\
\hline $\begin{array}{l}\text { The Chicago Manual of } \\
\text { Style }\end{array}$ & Total & 3 \\
\hline \multirow[t]{2}{*}{ Vancouver (UMR) } & Ciencias de la Salud & 28 \\
\hline & Psicología y Educación & 1 \\
\hline \multirow[t]{2}{*}{ Vancouver (UMR) } & Total & 29 \\
\hline & Total & 137 \\
\hline
\end{tabular}

Se verifica que las normas APA, Vancouver (UMR) e ISO-690 son las de mayor profusión y uso entre las revistas científicas objeto del estudio, y que entre las tres superan el $83 \%$ del total, si bien es APA la más utilizada. En este aspecto no parece haber gran diferencia entre el contexto de la revista electrónica y el de la revista impresa, si bien esto puede variar por el tema de la preservación digital.

Algunas publicaciones comienzan a exigir el uso de sistemas de almacenamiento de referencias bibliográficas como Webcite ${ }^{12}$ (http://www.webci tation.org) o $\mathrm{CitEc}^{13}$ (http://citec.repec.org) para garantizar así el acceso a

12 Es un portal que puede ser utilizado por los autores de los artículos o los editores de las revistas científicas en línea para generar una referencia permanente al contenido del cual se almacena una copia de seguridad por si se llegara a perder el contenido original, algo no del todo infrecuente en las revistas y sitios académicos.

13 Portal que permite el análisis de citas de los documentos de la biblioteca digital RePEc. Para cada documento puesto a disposición en formato electrónico se extraen automáticamente y analizan sus listas de referencias. De esta forma sabemos que son documentos que han sido citados, pero ¿cuántas veces y cuáles son los documentos de donde parten esas citas? 
la fuente informativa. Estos sistemas proporcionan, entre otras cosas, las referencias elaboradas según un estilo internacional. Esto puede terminar incidiendo en la política editorial de las revistas y propiciar un uso masivo de un estilo o norma adoptados por estos sistemas de almacenamiento para la preservación digital.

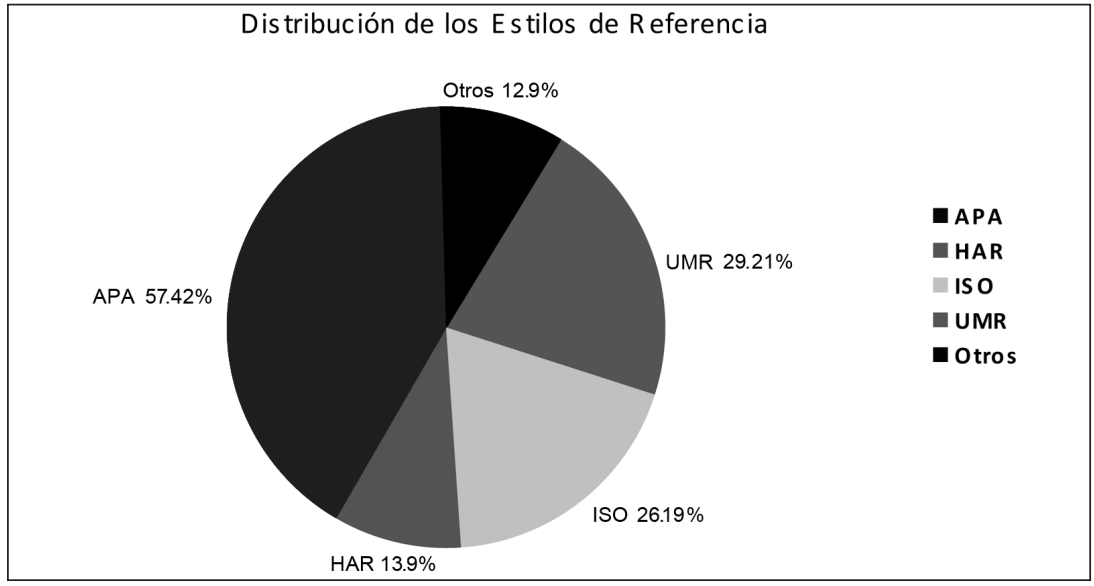

Figura 9. Distribución de los estilos de referencia bibliográfica empleados en las revistas analizadas.

En la Tabla III se recogen datos que relacionan las áreas científicas analizadas con la norma específica más utilizada en ese campo del conocimiento (no es una tabla exhaustiva, quedan fuera de ellas las relaciones de menor frecuencia). En otras áreas no se detectan vínculos de similar fortaleza.

Tabla III. Estilos de referencia bibliográfica más empleados en las revistas españolas OAl según la materia.

\begin{tabular}{|l|l|c|}
\hline \multicolumn{1}{|c|}{ Materia } & \multicolumn{1}{c|}{ Estilo } & Núm. \\
\hline Psicología y Educación & APA & 37 \\
\hline Ciencias de la Salud & Vancouver (UMR) & 28 \\
\hline Ciencias Sociales & IS0 690 / APA & 11 \\
\hline Economía y Empresa & IS0 690 / APA & 4 \\
\hline Humanidades & IS0 690 / IS0 690-2 & 3 \\
\hline Filologías & APA & 3 \\
\hline Tecnologías & APA & 2 \\
\hline Geociencias, Medio Ambiente & IS0 690 / IS0 690-2 & 2 \\
\hline Ciencias Jurídicas & IS0 690 & 2 \\
\hline Ciencias Biológicas & Harvard / IS0 690 & 2 \\
\hline Arte & APA / Harvard & 1 \\
\hline
\end{tabular}




\section{Formato de la cita}

La Tabla IV recoge el formato de cita empleado en todas y cada una de las revistas analizadas agrupadas por las materias.

Tabla IV. Formatos de cita más empleado en las revistas españolas OAl agrupados según materia.

\begin{tabular}{|c|c|c|}
\hline Formato & Materia & Núm. \\
\hline \multirow[t]{9}{*}{ | Apellidos, año | } & Arte y Humanidades & 6 \\
\hline & Biología, Geociencias, Medio Ambiente & 5 \\
\hline & Ciencias de la Salud & 2 \\
\hline & Ciencias Jurídicas & 1 \\
\hline & Ciencias Sociales & 20 \\
\hline & Economía y Empresa & 6 \\
\hline & Filologías & 5 \\
\hline & Psicología y Educación & 38 \\
\hline & Total & 83 \\
\hline \multirow[t]{9}{*}{ | Apellidos, año, página | } & Arte y Humanidades & 3 \\
\hline & Biología, Geociencias, Medio Ambiente & 2 \\
\hline & Ciencias Jurídicas & 3 \\
\hline & Ciencias Sociales & 8 \\
\hline & Economía y Empresa & 3 \\
\hline & Filologías & 1 \\
\hline & Psicología y Educación & 1 \\
\hline & Tecnologías & 3 \\
\hline & Total & 24 \\
\hline \multirow[t]{5}{*}{ | Numérico | } & Arte y Humanidades & 1 \\
\hline & Ciencias de la Salud & 28 \\
\hline & Psicología y Educación & 1 \\
\hline & Total & 30 \\
\hline & Total & 137 \\
\hline
\end{tabular}

Con gran diferencia (más de dos tercios del total), el formato de cita más empleado es | Apellidos, año I. Esto concuerda con la lógica de la revista electrónica donde muchas de ellas han llegado incluso a suprimir el número de página (especialmente aquellas que publican los artículos en formato HTML o XHTML). Si bien el contexto del acceso abierto está introduciendo de forma paulatina muchos cambios en los procesos de edición de revistas científicas, se siguen conservando algunas tradiciones, como que las revistas de Ciencias de la Salud utilizan mayoritariamente la citación numérica. Estos resultados coinciden con lo planteado por Muñoz Alonso:

[...] el sistema de citación que numerosos investigadores eligen para la presentación de los originales es el sistema de autor-año, sistema muy recomendado por 
determinados manuales de estilo de las universidades norteamericanas o de ámbito anglosajón, sobre todo para la mayoría de las ciencias sociales y las ciencias naturales, si bien también se está imponiendo este sistema en el campo de las ciencias humanas, sobre todo por la distinción que permite entre citas, notas y referencias bibliográficas. (2006: 97)

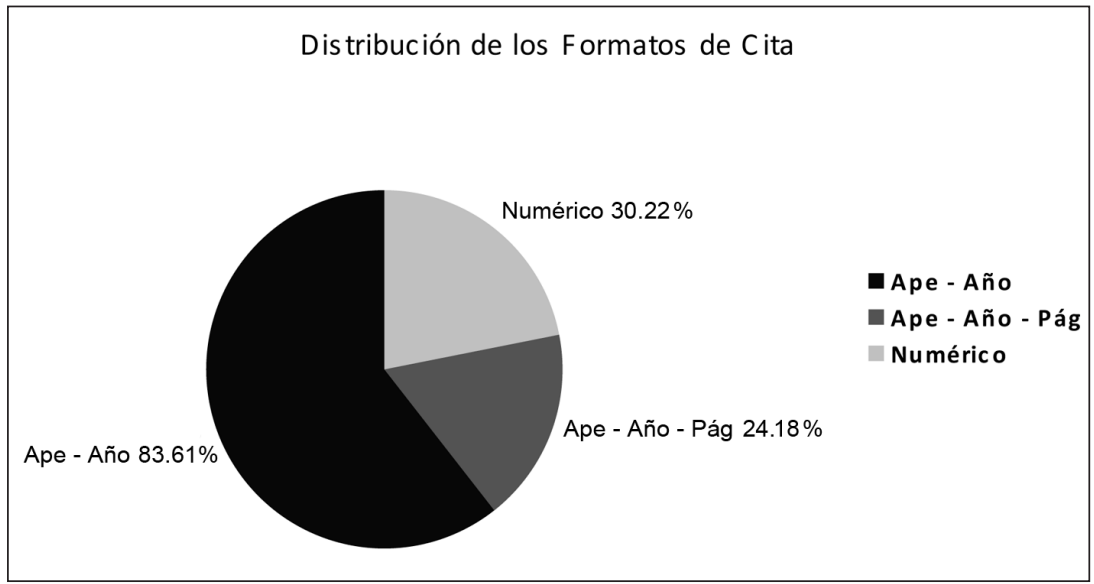

Figura 10. Distribución de los formatos de cita empleados en las revistas analizadas.

\section{Consideraciones finAles}

Si bien el número de publicaciones españolas presentes en el directorio DOAJ es considerable, no se puede afirmar que la normalización bibliográfica esté muy extendida entre ellas, ya que sólo un tercio la imponen a sus autores como requisito de publicación. Tampoco parece que los repertorios internacionales valoren este requisito a la hora de evaluar la inclusión o no de estas revistas, si bien esto puede deberse a que sea el editor de la revista quien lleve a cabo esa normalización bibliográfica, algo complicado de entender porque adiciona un tiempo de trabajo suplementario que puede llegar a ser extenso y que suele provocar dilaciones excesivas en la publicación de los documentos, aún más en este contexto donde esta actividad editorial se lleva a cabo sin ánimo de lucro alguno y casi sin presupuestos. En todo caso, los repositorios digitales científicos basados en el uso del protocolo OAI-MPH, destino lógico de esta producción científica, agradecerían, sin duda alguna, un mayor nivel de normalización.

La colección analizada presenta asimismo una serie de desequilibrios por áreas de conocimiento, concentrándose gran parte de la producción en las 
áreas de Psicología, Educación, Ciencias Sociales (en general) y Ciencias de la Salud, siendo destacable la escasa presencia de áreas tecnológicas. El predominio de estas áreas conlleva a que el estilo de referencia bibliográfica más empleado sea el APA (mayoritario en Psicología y Ciencias Sociales) y, en segundo lugar, el formato Vancouver (muy presente en Ciencias de la Salud) debido a la gran popularidad de estos estilos en estos campos del conocimiento a los que seguramente ayuda el hecho de su gratuidad de acceso y consulta.

La forma de citar más empleada es IApellidos, añol; algo consecuente, en nuestra opinión, con el contexto de las revistas electrónicas donde el número de página es un elemento en proceso de extinción en muchos casos. Esto no implica que se pierdan algunas tradiciones, se han identificado fuertes asociaciones entre materias, formatos y estilos que también se producen en la clásica edición impresa. La asociación más diáfana se presenta entre las revistas de Ciencias de la Salud, la norma Vancouver y el formato de cita numérico; ocurriendo algo similar en las revistas de Psicología y Educación, la norma APA y las citas IApellidos, añol.

Por último queremos destacar que en la realización del trabajo se ha detectado la ausencia en la muestra de algunas revistas científicas de cierto prestigio en sus respectivas áreas de conocimiento. La causa a la que puede achacarse esta ausencia es su no inclusión en el DOAJ porque la revista impone algún tipo de restricción al acceso abierto (como introducir algún tipo de embargo o periodo reservado a suscriptores). Postulamos a favor de que estas publicaciones supriman tales limitaciones, ya que los porcentajes de revistas normalizadas, frente a las no normalizadas, aumentarían la presencia en repertorios internacionales y se favorecería la inmediatez en el acceso al conocimiento.

\section{BiBLIOGRAFÍA}

Abadal, E. (2012), "Retos de las revistas en acceso abierto: cantidad, calidad y sostenibilidad económica”, en Hipertext.net, núm. 10. Disponible en: http://www.upf.edu/hipertextnet/numero-10/retos-revistas-en-acceso-abierto.html [Fecha de consulta: 28 de diciembre de 2012].

Anglada i de Ferrer, L.; Ros Gorné, R. y Reoyo Tudó, S. (2005), “Los repositorios como componentes esenciales de las bibliotecas digitales: la experiencia de las bibliotecas universitarias de Cataluña (CBUC)", en III Jornada sobre la Biblioteca Digital Universitaria. Disponible en: http://www.recercat.net/bitstream/2072/1167/1/ 0510JBDUArgentina.pdf [Fecha de consulta: 28 de diciembre de 2012]. 
Álvarez De Toledo, L. (2012), Principales estilos de citas bibliográficas: Infobiblio [mensaje en blog], 18 de junio. Disponible en: http:// infobiblio.es/principales-estilos-de-citas-bibliograficas [Fecha de consulta: 28 de diciembre de 2012].

Angrosh, M. A.; Cranefield, S. y Stanger, N. (2011), "Contextual information retrieval in research articles: Semantic publishing tools for the research community", en Information Science Discussion Papers Series, núm. 2011/06. Disponible en: http://hdl.handle. net/10523/1783 [Fecha de consulta: 28 de diciembre de 2012].

Benz, D. et al. (2009), "Managing Publications and Bookmarks with BibSonomy. Managing publications and bookmarks with BibSonomy”, en Ciro Cattuto, Giancarlo Ruffo y Filippo Menczer (eds.), HT '09: Proceedings of the 20th ACM Conference on Hypertext and Hypermedia, ACM, New York, NY, USA, pp. 323-324. Disponible en: http://www.kde.cs.uni-kassel.de/pub/pdf/benz2009managing. pdf [Fecha de consulta: 28 de diciembre de 2012].

Benz, D.; Körner, C.; Hotho, A.; Stumme, G. y Strohmaier, M. (2011), "One tag to bind them all: measuring term abstractness in social metadata”, en Antonioiu et al. (eds.), ESWC 2011. Part II (LNCS, vol. 6644), pp. 360-374.

Björk, B-C.; Welling, P.; Lakso, M.; Majlender, P.; Hedlund, T. y Guönason, G. (2010), "Open Access to the Scientific Journals Literature: situation 2009”, en PloS ONE vol. 5, núm. 6. DOI:10.1371/ journal.pone.0011273 [Fecha de consulta: 29 de diciembre de 2012].

Culebras Fernández, J. (2010), Utilidad y contemporaneidad de las referencias bibliográficas en los artículos científicos. Disponible en: http://www.jano.es/ficheros/sumarios/1/0/1763/82/00820087_LR. pdf [Fecha de consulta: 08 de enero de 2013].

Delgado López-Cózar, E. (1997a), "Evaluación del grado de ajuste de las revistas españolas de ciencias de la salud a las normas internacionales de presentación de publicaciones periódicas", en Revista Española de Salud Pública, vol. 71, núm. 6, pp. 531-546. Disponible en: http://scielo.isciii.es/scielo.php?pid=S1135-572719970006000 03\&script=sci_arttext [Fecha de consulta: 07 de enero de 2013].

- (1997b), "Evaluación y aplicación de las normas de presentación de publicaciones periódicas: revisión bibliográfica”, en Revista Española Documentación Científica, vol. 20, núm. 1, pp. 39-51. Disponible en: http://redc.revistas.csic.es/index.php/redc/article/view Article/599 [Fecha de consulta: 26 de diciembre de 2012].

Gámez, M. (2013), "La evaluación de revistas de psicología: correlación entre el factor de impacto, el índice $b$ y los criterios de Latindex”, en Investigación Bibliotecológica, vol. 27, núm. 61, septiembre/ diciembre, pp. 15-27. Disponible en: http://www.revistas.unam. mx/index.php/ibi/article/view/42812 [Fecha de consulta: 13 de enero de 2014]. 
Hernández Pina, F. y Maquilón Sánchez, J. J. (2010), "Indicadores de calidad de las revistas científicas y sistema de gestión editorial mediante OJS”, en Anales de Documentación, vol. 28, núm. 1, pp. 13 29. Disponible en: http://revistas.um.es/rie/article/view/109941 [Fecha de consulta: 07 de enero de 2013].

Harnad, S.; Brody, T.; Vallieres, F.; Carr, L.; Hitchcock, S.; Gingras, Y.; Oppenheim, C.; Hajjem, C. y Hilf, E. R. (2008), “The Access/Impact Problem and the Green and Gold Roads to Open Access: An Update”, en Serials Review, vol. 34, núm. 1, pp. 36-40. DOI: 10.10 16/j.serrev.2007.12.005 [Fecha de consulta: 10 de enero de 2014].

Iniciativa de Budapest para el Acceso Abierto (2002). Disponible en: http: //www.budapestopenaccessinitiative.org/translations/spanish-trans lation [Fecha de consulta: 07 de febrero de 2013].

Jacso, P. (2005), "As we may search - Comparison of major features of the Web of Science, Scopus, and Google Scholar citation-based and citation-enhanced databases", en Current Science, vol. 89, núm. 9, pp. 1537-1547. Disponible en: http://choo.fis.utoronto.ca/ FIS/courses/LIS1325/Readings/jacso.pdf [Fecha de consulta: 07 de enero de 2013].

Kovalchik, S. (2012), Google starts ranking journals [mensaje de blog]. Disponible en: http://www.significancemagazine.org/details/we bexclusive/1788885/Google-starts-ranking-journals.html [Fecha de consulta: 07 de enero de 2013].

Laakso, M.; Welling, P.; Bubvova, H.; Nyman, L.; Börjk, B-C. y Hedlund, T. (2011), "The development of OAJ publishing from 1993 to 2009”, en Plos ONE, vol. 6, núm. 6. DOI: 10.1371/journal.pone, 0020961 [Fecha de consulta: 27 de diciembre de 2013].

Muñoz-Alonso, G. (2006), "Tendencias actuales de citación en los trabajos de investigación filosófica”, en Investigación Bibliotecológica, Instituto de Investigaciones Bibliotecológicas y de la Información/UNAM, vol. 20, núm. 41, pp. 91-106. Disponible en: http: //www.ejournal.unam.mx/ibi/vol20-41/IBI002004104.pdf [Fecha de consulta: 07 de enero de 2013].

NISO (2004), Understanding metadata. Disponible en: http://www.ni so.org/publications/press/UnderstandingMetadata.pdf [Fecha de consulta: 07 de enero de 2013].

Noruzi, A. (2005), "Google Scholar: the new generation of citation indexes”, en Libri, vol. 55, pp. 170-180. Disponible en: http://www. librijournal.org/pdf/2005-4pp170-180.pdf [Fecha de consulta: 07 de enero de 2013].

Ostrowska, A. (2009), “Open Access Journals Quality - How to Measure It?", en INFORUM 2009: 15th Conference on Professional Information Resources. Disponible en: http://www.inforum.cz/pdf /2009/ostrowska-aneta-eng.PDF [Fecha de consulta: 26 de diciembre de 2012]. 
Pérez-Borges, A. (2010), "Empleo de citas y referencias bibliográficas en trabajos científicos", en Documentación de las Ciencias de la Información, vol. 33, pp. 185-193. Disponible en: http://revistas.ucm. es/inf/02104210/articulos/DCIN1010110185A.PDF [Fecha de consulta: 07 de enero de 2013].

Pérez Solís, D. y Rodríguez Fernández, L. M. (2005), "El acceso abierto a la información científica", en Boletín de la Sociedad de Pediatría de Asturias, Cantabria y Castilla y León, vol. 45, pp. 6164. Disponible en: https://www.sccalp.org/documents/0000/10 37/BolPediatr2005_45_061-064.pdf [Fecha de consulta: 07 de enero de 2013].

Poynder, R. (2008), Open Access: The question of quality [mensaje en blog], 21 de noviembre. Disponible en: http://poynder.blogspot. com.es/2008/11/open-access-question-of-quality_21.html [Fecha de consulta: 26 de diciembre de 2012].

Public Knowledge Project (1998). Disponible en: http://pkp.sfu.ca/ about [Fecha de consulta: 20 de diciembre de 2012].

Recolecta (2010), Guí para la evaluación de repositorios institucionales de investigación, Madrid, Proyecto Recolecta. Disponible en: http://digital.csic.es/bitstream/10261/35735/1/GuiaEvaluacionRe colectav1.0-1.pdf [Fecha de consulta: 11 de enero de 2013].

Ruiz-Pérez, R.; Delgado López-Cózar, E. y Jiménez-Contreras, E. (2006), "Criterios del Institute for Scientific Information para la selección de revistas científicas: su aplicación a las revistas españolas: metodología e indicadores", en International Journal of Clinical and Health Psycology, vol. 6, núm. 2, pp. 401-424. Disponible en: http://ec3.ugr.es/publicaciones/Criterios_del_Institute_for_ Scientific_Information_para_la_seleccion_de_revistas_cientificas. pdf [Fecha de consulta: 07 de enero de 2013].

Ruiz Pérez, S. (2010), La edición de revistas científicas en acceso abierto: características editoriales y modelos de negocio en el contexto del Proyecto SOAP (Study of Open Access Publisbing), trabajo de fin de máster, España, Universidad de Granada, Facultad de Comunicación y Documentación. Disponible en: http://eprints.rclis. org/14951/1/MasterThesis-SergioRuiz.pdf [Fecha de consulta: 12 de enero de 2014].

Solaro, G. N. y Lamberti, A. M. (2011), Open Access: un nuevo camino para recorrer en la sociedad del conocimiento. Disponible en: http: //eprints.rclis.org/17265/ [Fecha de consulta: 07 de enero de 2013].

Sorli Rojo, A. y Merlo Vega, J. A. (2000), "Estilos de citas y referencias de documentos electrónicos", en Revista Española de Documentación Científica, vol. 23, núm. 4. Disponible en: http://digital.csic.es/hand le/10261/9457 [Fecha de consulta: 07 de enero de 2013].

Valencia Velázquez, D. I. (2013), Software profesional para construir repositorios digitales, tesis, Universidad Nacional Autónoma de México, Facultad de Ingeniería. Disponible en: http://eprints.rclis. org/19873/1/Tesis.pdf [Fecha de consulta: 08 de enero de 2014]. 
Villamón, M.; Devís, J. y Valenciano, J. (2006), “Análisis de las instrucciones para autores de las revistas españolas de ciencias de la actividad física y el deporte", en Motricidad: European Journal of Human Movement, vol. 16, pp. 133-150. Disponible en: http://revistamotricidad.es/openjs/index.php?journal $=$ motricidad $\&$ page $=$ article\&op=view \&path\%5B\%5D=142 [Fecha de consulta: 07 de enero de 2013].

Zurita Sánchez, J. M.; Cervantes Cruz, A. y Miranda Quevedo, P. (2007), "Propuesta de normalización bibliográfica para la creación de metadatos basados en Dublin Core en los Repositorios Universitarios del proyecto 3R", en II Encuentro Nacional de Catalogación "En los umbrales de un nuevo código de catalogación". Disponible en: http://eprints.rclis.org/10594/ [Fecha de consulta: 11 de enero de 2013]. 